Artículo Original

\title{
Estado de salud periodontal de gestantes después del uso de una pasta dental con extractos vegetales
}

Status of periodontal health in pregnant women after using a toothpaste with herbal extracts

\section{Resumen}

Existe una alta prevalencia de problemas gingivales ampliamente comprobada en gestantes. Este estudio tuvo como propósito determinar el estado de salud periodontal de gestantes después del uso de una pasta dental con extractos vegetales. Participaron 80 pacientes gestantes del Centro Médico Naval "Cirujano Mayor Santiago Távara” en un estudio clínico cuasi experimental, prospectivo y longitudinal. Se dividió la muestra en dos grupos dando a cada paciente un cepillo y una pasta dental; en el grupo experimental, se entregó una pasta dental con extractos vegetales y en el control una pasta dentífrica convencional. Se examinó la gravedad de inflamación gingival, placa bacteriana y hemorragia gingival al inicio del estudio y luego de 28 días. Se observó una mejora estadísticamente significativa del estado periodontal en ambos grupos siendo mayor la mejora en el grupo experimental, la diferencia entre ambos grupos fue significativa en inflamación gingival y placa bacteriana. Al comparar el efecto según los trimestres de gestación, la pasta con extractos vegetales tuvo similar efectividad que el control en el tercer trimestre. Se concluye que la pasta dental con extractos vegetales redujo la enfermedad periodontal en gestantes sin ser consistentemente mejor que el control; su efecto no fue superior a la pasta convencional en el tercer trimestre de gestación.

\begin{abstract}
The correlation between gum disease and pregnancy is highly proven. The aim of this study was to determine the status of periodontal health in pregnant women after using toothpaste with herbal extracts. 80 pregnant women of the Naval Medical Center "Cirujano Mayor Santiago Távara" participated in this prospective, longitudinal, quasi-experimental clinical study. The sample was divided in two groups, the experimental group received toothpaste with herbal extracts and the control group conventiona toothpaste, both groups received a toothbrush. Data collected at baseline and after 28 days included severity of gingival inflammation, dental plaque and gingival bleeding. A significant reduction of periodontal disease was found in both groups, the experimental group had a better effect than control group. Differences between the groups were significant in gingival inflammation and dental plaque. The herbal toothpaste had a similar effect than conventional in the third trimester. The herbal toothpaste reduced periodontal disease in pregnant women without being consistently better than control. A certain reduction of herbal toothpaste's effect in the third trimester is suggested.
\end{abstract}

\section{Introducción}

Las enfermedades periodontales afectan a gran parte de la población mundial ${ }^{1}$. Se relacionan con la presencia de placa dental así como a condiciones sistémicas. ${ }^{2} \mathrm{La}$ mujer durante el embarazo sufre grandes cambios que la predisponen a presentar gingivitis gestacional..$^{3-6}$ Estos problemas se asocian a placa bacteriana, por esto es de gran importancia el control de la higiene oral durante la gestación.?

El tratamiento de la enfermedad periodontal se centra en la eliminación y control de la placa bacteriana. ${ }^{8}$ El método más utilizado es el cepillado con pasta dental, pero debido a la deficiencia en la técnica de la mayoría la composición de los dentífricos, es constantemente, mejorada. Una pasta dental $(\text { Parodontax })^{\oplus}$ con extractos vegetales (ratania, equiná- cea, mirra, manzanilla y salvia), bicarbonato de sodio y flúor tiene estudios contradictorios sobre su efecto. El objetivo de la presente investigación fue determinar la eficacia de una pasta dental con extractos vegetales en el estado de salud periodontal de gestantes; además, de observar el efecto de dicha pasta según el trimestre de embarazo.

\section{Métodos}

En el presente estudio clínico cuasi experimental, prospectivo y longitudinal, la población fueron las pacientes gestantes del CEMENA (Centro Médico Naval) "Cirujano Mayor Santiago Távara" en un periodo de 2 meses. Se incluyeron 80 personas con: edad gestacional $<32$

\section{Daniel Alvaro Coronado Tamariz ${ }^{1}$ Sixto García Linares ${ }^{2}$}

Egresado de la Facultad de Odontología, Universidad Nacional Mayor de San Marcos, Lima, Perú.

Docente del Departamento Académico Médico-Quirúrgico de la UNMSM.

Correspondencia:

CD Daniel Alvaro Coronado Tamariz

E-mail: tcdan@hotmail.com

Palabras clave: Pasta dental herbal y terapia gingival, enfermedad periodontal, gestación, parodontax ${ }^{\odot}$ índice gingival, sangrado gingival al sondaje, índice de placa dental.
Keywords: Herbal Toothpaste and Gum Therapy, Periodontal disease, Pregnancy, Parodontax ${ }^{\oplus}$, Gingival index, Gingival Bleeding on Probing, Dental Plaque Index. semanas según Fecha de Última Regla (FUR) y $\geq 20$ piezas en boca. Los criterios de exclusión fueron: enfermedad sistémica, alergia a pastas usadas, riesgo o complicación del embarazo, recibir terapia antibiótica o antiinflamatoria y usar ortodoncia. Primero se entrevistó al paciente para determinar si cumplía los criterios de inclusión, luego se detalló la investigación y firmó el consentimiento informado. El estudio se hizo respetando la Declaración de Helsinki. ${ }^{10}$

A cada paciente se le colocó en un grupo al azar, teniendo 40 sujetos en cada uno (experimental y control). Se examinaron los índices gingival (IG) de Löe, ${ }^{11}$ de placa bacteriana (IP) de Silness y Löe. ${ }^{12}$ y de sangrado gingival (ISG) de Muhlemann y Son ${ }^{13}$ utilizando una 
sonda periodontal Maryland marca Hu-Friedy ${ }^{\oplus}$. Se evaluaron las 4 caras de cada pieza evaluada, considerando en el IG e IP las piezas: 1,$6 ; 2,1 ; 2,4 ; 3,6 ; 4,1$ y 4,$4 ;{ }^{14}$ en el ISG se valoraron las piezas anteriores. Las participantes recibieron un cepillo dental de cerdas suaves y planas, cabeza pequeńa y rectangular. El grupo experimental recibió un tubo de Parodontax Flúor ${ }^{\oplus}(70 \mathrm{~g})$ y el control una pasta convencional (Dento ${ }^{\circ}$ ) de $124 \mathrm{~g}$ con carbonato de calcio. Las pacientes se cepillaron 3 veces al día y no utilizaron otro método de higiene. No se realizó profilaxis ni se modificó la técnica de cepillado al inicio del estudio. Luego de 28 días, se realizó el control de los índices, se informó a las pacientes su estado de salud oral y se enseñó la técnica de cepillado (Bass) e hilo dental.

Los resultados se procesaron con el programa SPSS ${ }^{\oplus 15}$. Los índices fueron promediados realizando tablas y gráficos. Para comparar las medias entre ambos grupos, se usó la prueba $T$ para muestras independientes y para comparar el índice inicial y final de cada grupo la prueba $\mathrm{T}$ para muestras relacionadas $(\alpha=0,05)$.

\section{Resultados}

Siendo la muestra 80 pacientes, hubo $48,75 \%$ de [27-33], 32,5\% de [3440] y $18,75 \%$ de [19-26] años. La muestra fue de multigestas en su mayoría $(83,75 \%)$. Cursaban el primer trimestre $22,5 \%$, el segundo $43,75 \%$ y el tercer $33,75 \%$. Finalizaron el estudio 73 pacientes, tres no usaron la pasta experimental por náuseas, tres tuvieron abortos y unas complicaciones en el embarazo.

En los índices iniciales no hubo diferencias significativas entre los grupos. $\mathrm{Al}$ comparar los índices iniciales con los finales en cada grupo se halló una reducción altamente significativa $(\mathrm{p}<0,01)$ del IG, IP e ISG. Los índices finales del grupo experimental fueron significativamente menores que el control, con valores $p$ de $\mathrm{IG}=0,010$ e $\mathrm{IP}=0,003$. En el ISG esta diferencia no fue significativa $(\mathrm{p}=0.203)$.

Según el trimestre de gestación, hubo una reducción significativa de todos los índices en ambos grupos, excepto en el ISG en el $1^{\circ}$ trimestre. Los índices finales del grupo experimental fueron menores a los del control, diferencia significativa en el IG: solo primer trimestre ( $p=0,02)$; IP: primer $(\mathrm{p}=0,025)$ y segundo trimestre $(\mathrm{p}=0,016)$; e ISG: en ningún trimestre.

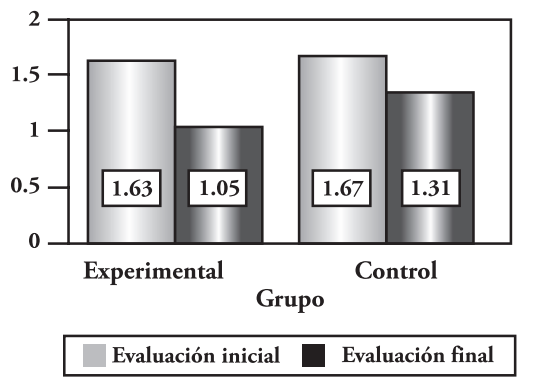

Fig 1. Inflamación gingival inicial y final luego del uso de pasta dental con/sin extractos vegetales.

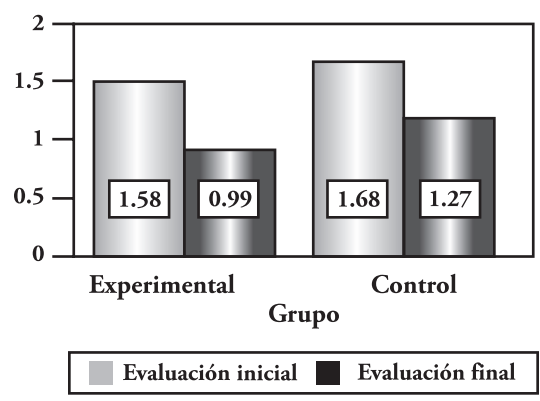

Fig 2. Placa bacteriana inicial y final luego del uso de pasta dental con/sin extractos vegetales.

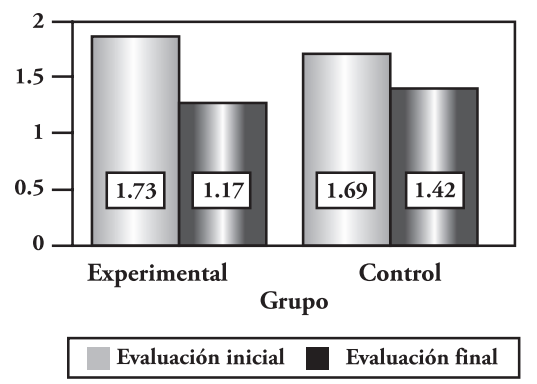

Fig 3. Sangrado gingival inicial y final luego del uso de pasta dental con/sin extractos vegetales.

\section{Discusión}

En el presente estudio, se observó un mejor efecto de la pasta con extractos vegetales que el control sobre inflamación gingival y placa bacteriana, mas no sobre hemorragia gingival. Yankell, ${ }^{15}$ usando también una pasta con carbonato de calcio como control, halló un mejor efecto significativo de la pasta experimental en IP e ISG (pero no en IG), probablemente debido al mayor tiempo de uso (6 meses). Saxer ${ }^{16}$ halló un mejor efecto significativo de la pasta experimental que una convencional sobre ISG en 28 días; sin embargo, el dentífrico control no disminuyó la hemorragia, razón probable de la diferencia entre los grupos.

No se encontraron diferencias en el efecto de las pastas sobre el ISG, probablemente porque los pacientes no usaron hilo dental. Graves ${ }^{17}$ comprobó el mejor efecto de cepillado con hilo que cepillado solo. Además, no se eliminó la placa y el cálculo al inicio ${ }^{18,19}$.

En el tercer trimestre ambas pastas tuvieron efectos similares, probablemente por los mayores problemas periodontales que ocurren en esta etapa $\mathrm{a}^{11,20-23}$.

Otros autores encontraron que la pasta Parodontax no redujo el IP e IG. Guggenheim $^{24}$ no halló efecto en ratas, sin embargo la evaluación de placa en estas es de poca confiabilidad. Pannuti ${ }^{25}$ no encontró diferencias entre la pasta experimental y control, pero en una muestra con índices bajos, realizando profilaxis y fisioterapia inicial.

\section{Conclusiones}

La pasta herbal es efectiva en la reducción de la enfermedad periodontal en gestantes. Fue mejor que el control, sin serlo consistentemente en la reducción de hemorragia gingival. Se justifica su uso sobre las pastas convencionales. En el tercer trimestre, la pasta no demostró ser superior al dentífrico control.

\section{Agradecimiento}

A Glaxo Smith Kline Perú S.A. por brindar las pastas dentales Parodontax ${ }^{\odot}$ y a Colgate-Palmolive Perú SA por facilitar los cepillos utilizados en el estudio.

\section{Referencias bibliográficas}

1. Newman M, Takei H, Carranza F. Carranza, Periodontología clínica, 9na ed. México: McGraw-Hill; 2004.

2. Lindhe J. Periodontología clínica e implantología odontológica. 4ta ed. Buenos Aires: Médica Panamericana; 2005.

3. Rose LF, Genco RJ, Cohen W, Mealey BL. Periodontal medicine. Hamilton, Canada: B.C. Decker Inc.; 2000.

4. Moreno E, Xiong X, Buekens P, Padilla N. Prevalencia de enfermedad periodontal en primigestas en un Centro de Salud Urbano en Celaya, Guanajuato. Revista ADM 2008; 65(1):13-19.

5. Cunningham FG. Williams Obstetrícia. 21 a ed. Buenos Aires: Médica Panamericana; 2002.

6. International Workshop for a Classification of Periodontal Disease and Conditions. The American Academy of Periodontology. 1999; 4(1). 
7. Little JW, Falace DA. Tratamiento odontológico del paciente bajo tratamiento médico. 5 ta ed. Barcelona: Elsevier; 1998.

8. Bravo M, Llodra J, Almerich J, Echeverría J. Control mecánico de placa. Actas del 1er Workshop Ibérico de control de placa e higiene buco-dental. Sociedad Española de Periodoncia y Osteointegración. Madrid; 2003.

9. Glaxo SmithKline México, 2006. Disponible en: http://www.gsk. com.mx/html/nuestros-productos/ cuidado-oral.html

10. World Medical Association. Declaration of Helsinki. JAMA 2008; 86(8).

11. Löe H, Silness J. Periodontal disease in pregnancy. I. Prevalence and severity. Acta Odontol Scand 1963; 21:533-51.

12. Silness J, Löe H. Periodontal disease in pregnancy. II. Acta Odontol Scand 1964; 22:121-35.

13. Muhlemann H, Son S. Gingival sulcus bleeding - a leading symptom in initial gingivitis. Helv Odontol Acta 1971; 15:107-113.

14. Ramfjord S. The periodontal disease index (PDI). J Periodontol 1967; 38:602-10.
15. Yankell SL, Emling RC, Perez B. Six-month evaluation of Parodontax dentifrice compared to a placebo dentifrice. J Clin Dent. 1993; 4(1):26-30.

16. Saxer U, Jaschouz V, Ley F. The effect of Parodontax dentifrice on gingival bleeding. J Clin Dent. 1994; 5(2):63-4.

17. Graves RC, Disney JA, Stamm JW. Comparative effectiveness of flossing and brushing in reducing interproximal bleeding. Journal of Periodontology 1989; 60(5):243-7.

18. Ribeiro É, Bittencourt S, NocitiJúnior F, Sallum E, Sallum A, Casati $M$. The effect of one session of supragingival plaque control on clinical and biochemical parameters of chronic periodontitis. J Appl Oral Sci 2005; 13(3):275-9.

19. Novaes A, Souza S, Taba M, Grisi M, Suzigan L, Tunes R. Control of gingival inflammation in a teenager population using ultrasonic prophylaxis. Braz Dent J 2004; 15(1):41-45.

20. Tanny D, Habashneh R, Hammad $\mathrm{M}$. The periodontal status of pregnant women and ist relationship sociodemografhic and clinical variables. Journal of Oral Rehabilitation 2003; 30:440-445.
21. Tremolada J. Prevalencia y severidad de gingivitis del embarazo y su correlación con las concentraciones de estradiol y progesterona [Tesis]. Lima: UNMSM; 1984

22. Martínez L, Salazar C, Ramírez G. Estrato social y prevalencia de gingivitis en gestantes. Estado Yaracuy, municipio San Felipe. Acta odontol. Venez. 2001 Ene; 39(1).

23. Machuca G, Khoshfeiz O, Lacalle JR, Machuca C, Bullon P. The influence of general health and sociocultural variables on the periodontal condition of pregnant women. Journal of periodontology 1999; 70:779-785.

24. Guggenheim B, Lutz F, Schmid R. Caries and plaque inhibition in rats by five topically applied dentifrices. Eur J Oral Sci 1997; 105:258-263.

25. Pannuti CM, Mattos JP, Ranoya PN, Jesus AM, Lotufo RFM, Romito GA. Clinical effect of a herbal dentifrice on the control of plaque and gingivitis. A double-blind study. Pesqui Odontol Bras 2003; 17(4):314-8.

Fecha de recepción: 5 de noviembre 2009

Fecha de aprobación: 5 de enero 2010 